\title{
GROWTH RATES OF DIMENSIONAL INVARIANTS OF COMPACT QUANTUM GROUPS AND A THEOREM OF HØEGH-KROHN, LANDSTAD AND STØRMER
}

\author{
CLAUDIA PINZARI \\ (Communicated by Marius Junge) \\ Dedicated to the memory of Claudio D'Antoni
}

\begin{abstract}
We give local upper and lower bounds for the eigenvalues of the modular operator associated to an ergodic action of a compact quantum group on a unital $C^{*}$-algebra. They involve the modular theory of the quantum group and the growth rate of quantum dimensions of its representations and they become sharp if other integral invariants grow subexponentially. For compact groups, this reduces to the finiteness theorem of Høegh-Krohn, Landstad and Størmer. Consequently, compact quantum groups of Kac type admitting an ergodic action with a non-tracial invariant state must have representations whose dimensions grow exponentially. In particular, $S_{-1} U(d)$ acts ergodically only on tracial $C^{*}$-algebras. For quantum groups with non-involutive coinverse, we derive a lower bound for the parameters $0<\lambda<1$ of factors of type $\mathrm{III}_{\lambda}$ that can possibly arise from the GNS representation of the invariant state of an ergodic action with a factorial centralizer.
\end{abstract}

\section{INTRODUCTION}

In the early 1980's Høegh-Krohn, Landstad and Størmer proved that the multiplicity of an irreducible representation of a compact group acting ergodically on a unital $C^{*}$-algebra is bounded above by its dimension and moreover the unique invariant state is a trace [7]. This result is often used as a finiteness criterion in operator algebras. Moreover, Wassermann, starting from this, showed the negative result that $\mathrm{SU}(2)$ acts ergodically only on type I von Neumann algebras [14].

If we consider compact quantum groups instead of compact groups, the ergodic theory on operator algebras becomes much richer. For example, as is well known, finiteness fails, as compact quantum groups may have non-involutive coinverse and, in this case, the Haar state has a non-trivial modular theory [15]. Boca generalized some of these results to ergodic actions of compact quantum groups. He proved that the quantum dimension is an upper bound for the multiplicity and that the invariant state satisfies the KMS property [3]. Wang has found many examples of ergodic actions of the free unitary quantum groups on factors of types II and III [13. In particular, his examples show that finiteness fails already in the case of compact quantum groups with involutive coinverse, often called of Kac type, even though these have trivial modular theory. Indeed, $A_{u}(n)$ can act ergodically on factors on type $\mathrm{III}_{\frac{1}{n}}$. Bichon, De Rijdt and Vaes have constructed actions of $A_{o}(F)$ with multiplicities larger than the integral dimensions [2. In joint papers with

Received by the editors February 15, 2011 and, in revised form, July 14, 2011.

2010 Mathematics Subject Classification. Primary 46L55, 46L65; Secondary 37A55, 28D20. 
Roberts, we have shown that any finite index inclusion of factors of type $\mathrm{II}_{1}$ gives rise to ergodic actions of $A_{o}(F)[10$. Moreover, the classification of ergodic actions of compact quantum groups is related to the classification of tensor $C^{*}$-categories with conjugates [11.

The aim of this paper is to show an analogue of the second part of the finiteness theorem of 7 for compact quantum groups.

The examples of [2], as well as subsequent developments of the general theory of ergodic actions 9], contributed to a further understanding of the invariant state. It became clear, for example, that it is always almost periodic in the sense of Connes [5], meaning that the associated modular operator is diagonal, and that its eigenvalues exhibit an explicit, separated, dependence on both the modular theory of the quantum group and the eigenvalues of certain strictly positive matrices canonically associated to the irreducible representations of the quantum group belonging in the spectrum of the action.

A consequence of this is that, in the special case where the invariant state of an action of a non-Kac type compact quantum group is factorial and has factorial centralizer, then the associated factor is necessarily of type $\mathrm{III}_{\lambda}$ with $0<\lambda \leq 1$ (cf. Sect. 3 for a more precise statement). Note that the invariant states of the mentioned examples of Wang satisfy this factoriality condition. Another class of examples has been recently studied by Vaes and Vergnioux 12. They considered the translation action of $A_{o}(F)$ over itself and they were able to prove, among other things, that if $F$ has rank at least 3 and satisfies a suitable condition, then the Haar state is factorial and moreover the factor generated by the GNS construction is full in the sense of Connes [5].

For a general ergodic action, the problem of studying modularity of the invariant state becomes that of studying the spectra of the associated matrices. In retrospect, the theorem of [7] amounts to showing that these matrices are always trivial in the classical case.

To study these spectra, we introduce two growth rates of dimensional invariants of the quantum group, that of integral dimensions, $\operatorname{Dim}_{u}$, and of quantum dimensions, $D_{u}$. These growth rates distinguish between $S U(2), S_{q} U(2)$ for $0<q<1$, $A_{o}(F)$, for $\operatorname{rank}(F) \geq 3$, and $A_{u}(F)$. More precisely, in the group case, quantum dimensions are just the integral dimensions, and therefore the growth rate is always polynomial, as a consequence of Weyl's dimension formula; hence $D_{u}=\operatorname{Dim}_{u}=1$ for all representations. This polynomial growth rate played an important role in the original proof of the finiteness theorem of [7].

Growth rate of quantum dimensions for $S_{q} U(2), 0<q<1$ is instead exponential. However, integral dimensions of irreducibles are the same as in the classical case; hence they grow polynomially. This is opposed to $A_{o}(F)$, for $\operatorname{rank}(F) \geq 3$, for which both growth rates are exponential. In the case of $A_{u}(F)$, both dimensional invariants of the fundamental representation have the largest possible growth rates as all tensor powers of this representation are irreducible.

We shall see that if the growth rate of the integral dimensions is subexponential, then that of the quantum dimensions, $D_{u}$, is explicitly determined by the eigenvalues of the modular operator of the quantum group (Prop. 4.3). However, this fact is not generally true, and $A_{o}(n)$, for $n \geq 3$, or $A_{u}(n)$, for $n \geq 2$, are the first examples. 
Given an ergodic action of a compact quantum group on a unital $C^{*}$-algebra, our main result consists of showing that $D_{u}$ and $D_{u}^{-1}$ are upper and lower bounds for the spectral radii of the mentioned matrices involved in the modular operator of the ergodic action (see Theorem 4.8). In particular, we reproduce the finiteness theorem of [7] from the fact that $D_{u}=1$ in the group case. Our bounds become equalities in the case of ergodic actions with high quantum multiplicities in the sense of [2], but integral multiplicities with subexponential growth rate.

We shall show that the invariant $D$ determines the parameter $\lambda=\frac{1}{n}$ of the Connes invariant $S$ in Wang's example with $A_{u}(n)$. In other words, the rate of growth of dimensions clarifies which compact quantum groups of Kac type can act on infinite factors. More precisely, one of the consequences of our result is that, among compact quantum groups of Kac type, only those having some irreducible representation whose integral dimension grows exponentially can possibly act on $C^{*}$-algebras with a non-tracial invariant state, and this explains Wang's examples. For example, all ergodic $C^{*}$-algebras for $S_{-1} U(d)$ are tracial.

For compact quantum groups with non-involutive coinverse, we derive a general lower bound for the possible parameters $0<\lambda<1$ of the type $\mathrm{III}_{\lambda}$ factors that can arise from the GNS representation of the invariant state, provided the centralizer algebra is a factor. This lower bound involves the modular theory of the quantum group, the growth rates $D_{u}$, and the spectrum of the action; see Cor. 4.13. We thus see that many parameters $\lambda$ may be excluded if some spectral information is known. For example, for $S_{q} U(2)$, with $0<|q|<1$, then $\lambda \geq|q|^{2 r}$, where $r$ labels the first spectral irreducible representation. For $A_{o}(F)$, with $F$ of rank $\geq 3$, then $\lambda \geq\left(\frac{q}{\|F\|^{2}}\right)^{r}$ where $q+\frac{1}{q}:=\operatorname{Trace}\left(F^{*} F\right)$ and $r$ is as before. As another example, $\lambda \geq \frac{\min \left\{q_{0}, q_{n}^{-1}\right\}}{\operatorname{Trace}\left(F^{*} F\right)}$, for $A_{u}(F)$, with $q_{0}$ and $q_{n}$ the smallest and largest eigenvalues of $F^{*} F$ if the fundamental representation is spectral.

Our proof relies on the duality theorem of [9], which allows a rather simple presentation.

This paper is organized as follows. Section 2 is devoted to the preliminaries: we recall the notion of standard solutions of the conjugate equations and the duality theorem for ergodic actions. In Section 3 we describe preliminary results on the modular theory of the invariant state. In Section 4 we discuss the main result and some corollaries.

\section{Preliminaries}

Standard solutions of the conjugate equations. We shall briefly recall the main features of tensor $C^{*}$-categories with conjugates [8]. Let $\mathcal{A}$ be a tensor $C^{*}$-category (always assumed to be strict, with irreducible tensor unit $\iota$, subobjects and direct sums). Arrows $R \in(\iota, \bar{u} \otimes u)$ and $\bar{R} \in(\iota, u \otimes \bar{u})$ are said to define a conjugate $\bar{u}$ of the object $u$ if they satisfy the conjugate equations

$$
\bar{R}^{*} \otimes 1_{u} \circ 1_{u} \otimes R=1_{u}, \quad R^{*} \otimes 1_{\bar{u}} \circ 1_{\bar{u}} \otimes \bar{R}=1_{\bar{u}} .
$$

If $R_{u}, \bar{R}_{u}$ and $R_{v}, \bar{R}_{v}$ are solutions for $u$ and $v$, then $R_{u \otimes v}:=1_{\bar{v}} \otimes R_{u} \otimes 1_{v} \circ R_{v}$, $\bar{R}_{u \otimes v}:=1_{u} \otimes \bar{R}_{v} \otimes 1_{\bar{u}} \circ \bar{R}_{u}$ are solutions for $u \otimes v$. Similarly, $R_{\bar{u}}:=\bar{R}_{u}, \bar{R}_{\bar{u}}:=R_{u}$ are solutions for $\bar{u}$. They are called the tensor product and conjugate solutions respectively. If conjugates exist for every object, then every object is the direct sum of irreducible objects. 
The category $\operatorname{Rep}(G)$ of (unitary, finite-dimensional) representations of a compact quantum group has conjugates and is embedded in the category of finitedimensional Hilbert spaces; hence arrows are linear maps. Solutions of the conjugate equations take the form $R=\sum_{i} j \psi_{i} \otimes \psi_{i}, \bar{R}=\sum_{k} j^{-1} \phi_{k} \otimes \phi_{k}$, with $j: H_{u} \rightarrow H_{\bar{u}}$ a unique invertible antilinear map between the representation Hilbert spaces and $\left(\psi_{i}\right),\left(\phi_{k}\right)$ orthonormal bases. Conjugate and tensor product solutions correspond to $j_{\bar{u}}:=j_{u}^{-1}, j_{u \otimes v}=j_{v} \otimes j_{u} \theta$, with $\theta: H_{u} \otimes H_{v} \rightarrow H_{v} \otimes H_{u}$ the flip map.

In a tensor $C^{*}$-category with conjugates it is convenient to select standard solutions of the conjugate equations, meaning that $\left\|R_{u}\right\|=\left\|\bar{R}_{u}\right\|$ if $u$ is an irreducible object, while if $u \simeq \bigoplus_{i} u_{i}$ with $u_{i}$ irreducible, $R_{u}:=\sum_{i} \bar{S}_{i} \otimes S_{i} \circ R_{u_{i}}$ $\bar{R}_{u}:=\sum_{i} S_{i} \otimes \bar{S}_{i} \circ \bar{R}_{u_{i}}$, where $R_{u_{i}}, \bar{R}_{u_{i}}$ are standard solutions and $\left\{S_{i} \in\left(u_{i}, u\right)\right\}$, $\left\{\bar{S}_{i} \in\left(\bar{u}_{i}, \bar{u}\right)\right\}$ are two sets of isometries whose ranges are pairwise orthogonal and add up to $1_{u}$ and $1_{\bar{u}}$, respectively. Clearly, the conjugate of a standard solution is standard. Standard solutions are unique up to unitary equivalence. Most importantly, they realize the minimal value of $\left\|R_{u}\right\|\left\|\bar{R}_{u}\right\|$ among all solutions, and they are characterized, up to scalars, by this property. This minimal value is the quantum (or intrinsic) dimension of $u$, denoted by $d(u)$. This implies the following fact, which will play a role.

Theorem 2.1 ([] ). The tensor product of standard solutions is standard.

We shall also need the following fact. In the category $\operatorname{Rep}(G)$, if $j_{u}: H_{u} \rightarrow H_{\bar{u}}$ defines a standard solution of the conjugate equations for $u, j_{u}^{*} j_{u}$ is a positive operator on $H_{u}$ which does not depend on the choice of the standard solution. This operator corresponds to $F_{u}^{-1}$ of Woronowicz [15]. It follows that the spectrum of $j_{u}^{*} j_{u}$ as well as its smallest and largest eigenvalues, denoted $\lambda_{u}$ and $\Lambda_{u}$ respectively, are invariantly associated to $u$ (in fact, to its equivalence class). Note that $\lambda_{\bar{u}}=$ $\Lambda_{u}^{-1}, \lambda_{u \otimes v}=\lambda_{u} \lambda_{v}$, and similarly for $\Lambda_{u \otimes v}$.

Ergodic actions, spectral functor and quasitensor functors. To a certain extent, we may think of the relationship between an ergodic action of a compact quantum group on a unital $C^{*}$-algebra, the associated spectral functor and an abstract quasitensor functor, as analogous to that between a Lie group, the associated Lie algebra and an abstract Lie algebra. The analogy is supported by the following properties of ergodic actions proved in [9] and is referred to as a duality theorem for ergodic actions. The spectral functor of an ergodic action is a quasitensor functor. For any ergodic action, there always is a maximal ergodic action which has the same spectral functor as that of the original one. It is the completion in the maximal $C^{*}$-norm of the dense spectral subalgebra of the given ergodic action. The maximal ergodic action with a given spectral functor is unique and canonically associated with it. Any abstract quasitensor functor from the representation category of $G$ to the Hilbert spaces is the spectral functor of an ergodic action. Two maximal ergodic actions of a given compact quantum group $G$ are conjugate if and only if the associated spectral functors are related by a unitary natural transformation. Hence quasitensor functors $\operatorname{Rep}(G) \rightarrow$ Hilb classify maximal ergodic $C^{*}$-actions of $G$.

In some more detail, let $G$ be a compact quantum group [16] and

$$
\alpha: \mathcal{C} \rightarrow \mathcal{C} \otimes \mathcal{Q}
$$


be an ergodic action of $G$ on a unital $C^{*}$-algebra $\mathcal{C}$ (i.e. $\mathcal{C}^{\alpha}:=\{c \in \mathcal{C}, \alpha(c)=$ $c \otimes I\}=\mathbb{C}) . \mathcal{Q}$ denotes the Hopf $C^{*}$-algebra of $G$. Let $u$ be a representation of $G$ on the Hilbert space $H_{u}$. Consider the space of linear maps $T: H_{u} \rightarrow \mathcal{C}$ intertwining $u$ with the action $\alpha$. The map taking $u$ to this space of intertwiners is a functor from the representation category of $G$ to the category of vector spaces. However, it is a contravariant functor; hence for convenience we consider the covariant functor obtained by passing to the category of dual vector spaces. This covariant functor will be denoted by $L$ (while we used the notation $\bar{L}$ in previous papers). As a consequence of ergodicity, the predual of $L_{u}$, and hence of $L_{u}$ itself, is a Hilbert space. We may make explicit $L_{u}=\left\{\sum_{i} \psi_{i} \otimes c_{i}, \quad \alpha\left(c_{i}\right)=\sum_{j} c_{j} \otimes u_{j, i}^{*}, \quad \psi_{i}\right.$ o. b. $\}$ and thus think of $L_{u}$ as the space of fixed points $\left(H_{u} \otimes \mathcal{C}\right)^{u \otimes \alpha}$ with inner product arising from the restriction of the $\mathcal{C}$-valued inner product of the free Hilbert module $H_{u} \otimes \mathcal{C}$. If $A \in(u, v)$ is an intertwiner in $\operatorname{Rep}(G)$, then $L_{A}$ acts as $A \otimes I$ from $L_{u}$ to $L_{v}$.

A ${ }^{*}$-functor $\mu: \mathcal{A} \rightarrow \mathcal{M}$ between two tensor $C^{*}$-categories is called quasitensor if there are isometries $\tilde{\mu}_{u, v} \in\left(\mu_{u} \otimes \mu_{v}, \mu_{u \otimes v}\right)$, such that

$$
\begin{gathered}
\mu_{\iota}=\iota, \\
\tilde{\mu}_{u, \iota}=\tilde{\mu}_{\iota, u}=1_{\mu_{u}}, \\
\tilde{\mu}_{u, v \otimes w}^{*} \circ \tilde{\mu}_{u \otimes v, w}=1_{\mu_{u}} \otimes \tilde{\mu}_{v, w} \circ \tilde{\mu}_{u, v}^{*} \otimes 1_{\mu_{w}}
\end{gathered}
$$

and a natural map in $u, v$,

$$
\mu(S \otimes T) \circ \tilde{\mu}_{u, v}=\tilde{\mu}_{u^{\prime}, v^{\prime}} \circ \mu(S) \otimes \mu(T),
$$

for objects $u, v, w, u^{\prime}, v^{\prime}$ of $\mathcal{A}$ and arrows $S \in\left(u, u^{\prime}\right), T \in\left(v, v^{\prime}\right)$. This definition was given in 9. Note that the most relevant axiom, (2.3), implies associativity: $\tilde{\mu}_{u, v, w}:=\tilde{\mu}_{u \otimes v, w} \circ \tilde{\mu}_{u, v} \otimes 1_{\mu_{w}}=\tilde{\mu}_{u, v \otimes w} \circ 1_{\mu_{u}} \otimes \tilde{\mu}_{v, w}$. If all the isometries $\tilde{\mu}_{u, v}$ are unitary, then $(2.3)$ is equivalent to associativity. In this case $(\mu, \tilde{\mu})$ will be called a relaxed tensor functor. Examples arise from pairs of non-isomorphic compact quantum groups with tensor equivalent representation categories. A well-known class of examples is $S_{\mu} U(2)$ and $A_{o}(F)$ for suitable conditions on $F$ (cf. Example 4.6). Composition of an equivalence $\operatorname{Rep}(G) \rightarrow \operatorname{Rep}\left(G^{\prime}\right)$ with the embedding functor of $\operatorname{Rep}\left(G^{\prime}\right)$ is a relaxed tensor functor $\operatorname{Rep}(G) \rightarrow$ Hilb.

For the spectral functor $L$ of an ergodic action, isometries making it a quasitensor are given by $\tilde{L}_{u, v}\left(\sum \psi_{i} \otimes c_{i}\right) \otimes\left(\phi_{j} \otimes d_{j}\right)=\sum\left(\psi_{i} \otimes \phi_{j}\right) \otimes d_{j} c_{i}$. Note that the spectral space of a non-spectral representation is trivial; hence, quasitensor functors, unlike the relaxed tensor ones, may take a non-zero object to the zero object.

A quasitensor functor $(\mu, \tilde{\mu})$ preserves conjugates, in the sense that if a non-zero object $u$ of $\mathcal{A}$ has a conjugate defined by arrows $R \in(\iota, \bar{u} \otimes u)$ and $\bar{R} \in(\iota, u \otimes \bar{u})$, then $\mu_{\bar{u}}$ is a conjugate of $\mu_{u}$, defined by $\hat{R}=\tilde{\mu}_{\bar{u}, u}^{*} \circ \mu(R), \hat{\bar{R}}=\tilde{\mu}_{u, \bar{u}}^{*} \circ \mu(\bar{R})$. This is a straightforward consequence of the axioms. The property of conservation of conjugates of a quasitensor functor $\mu$ implies $d\left(\mu_{u}\right) \leq\|\hat{R}\|\|\hat{\bar{R}}\| \leq d(u)$.

In particular, for the spectral functor of an ergodic action we may associate an antilinear invertible map $J_{u}: L_{u} \rightarrow L_{\bar{u}}$ to a solution $\left(R_{u}, \bar{R}_{u}\right)$ of the conjugate equations of a representation $u$ of $G$ by

$$
\sum_{k} J_{u} T_{k} \otimes T_{k}=\tilde{L}_{\bar{u}, u}^{*} \circ L\left(R_{u}\right)=\hat{R}_{u},
$$


where $T_{k}$ is an orthonormal basis of $L_{u}$, so that $\left\|\hat{R}_{u}\right\|^{2}=\operatorname{Trace}\left(J_{u}^{*} J_{u}\right)$ and $\left\|\hat{R}_{u}\right\| \leq$ $\left\|R_{u}\right\|$ with equality if $\tilde{L}_{\bar{u}, u}$ is unitary.

The above relation between intrinsic dimensions becomes a multiplicity bound of an irreducible representation in the spectum of the related ergodic action. Indeed, the scalar $\|\hat{R}\|\|\hat{\bar{R}}\|$ arising from the solution of the conjugate equations of an irreducible representation $u$, reduces to the quantum multiplicity $q-\operatorname{mult}(u)$ of [2], while the intrinsic dimension of $L_{u}$ is just the integral dimension of $L_{u}$, i.e. the ordinary multiplicity $\operatorname{mult}(u)$ of $u$ in the action. The above estimate says that $\operatorname{mult}(u) \leq \mathrm{q}-\operatorname{mult}(u) \leq d(u)$ a fact shown in 2 refining the inequality $\operatorname{mult}(u) \leq d(u)$ previously obtained by 3 . In the classical case, i.e. when $G$ is a compact group, the quantum dimension of a representation is just its integral dimension, and we thus in turn recover the classical result of Høegh-Krohn, Landstad and $\operatorname{Størmer}$ that $\operatorname{mult}(u) \leq \operatorname{dim}\left(H_{u}\right)$.

We shall need the following facts proved in 9 .

Lemma 2.2. Let $G$ be a compact quantum group acting ergodically on a unital $C^{*}$-algebra with spectral functor $(L, \tilde{L})$. If $J_{u}$ and $J_{v}$ are associated to solutions $j_{u}$ and $j_{v}$ of the conjugate equations for representations $u$ and $v$ of $G$ respectively, then

a) $J_{u \otimes v} \tilde{L}_{u, v}=\tilde{L}_{\bar{v}, \bar{u}} J_{v} \otimes J_{u} \Theta$, where $J_{u \otimes v}$ is associated to the tensor product solution for $u \otimes v$ and $\Theta: L_{u} \otimes L_{v} \rightarrow L_{v} \otimes L_{u}$ is the flip map;

b) for any $A \in(u, v), L\left(j_{v} A j_{u}^{-1}\right) J_{u}=J_{v} L(A)$.

Recall that conversely, given a quasitensor functor $(\mu, \tilde{\mu}): \operatorname{Rep}(G) \rightarrow$ Hilb, we may associate a maximal ergodic action of $G$ in the following way. Form the linear space ${ }^{\circ} \mathcal{C}_{\mu}:=\sum_{u \in \operatorname{Rep}(G)} \overline{\mu_{u}} \otimes_{\operatorname{Rep}(G)} H_{u}$, where $\otimes_{\operatorname{Rep}(G)}$ indicates a suitable tensor product treating the arrows of $\operatorname{Rep}(G)$ as scalars. The algebraic operations are defined by, dropping the indices and the natural transformation,

$$
(\bar{k} \otimes \psi)\left(\overline{k^{\prime}} \otimes \psi^{\prime}\right)=\left(\overline{k \otimes k^{\prime}}\right) \otimes\left(\psi \otimes \psi^{\prime}\right), \quad(\bar{k} \otimes \psi)^{*}=\overline{J k} \otimes j^{-1 *} \psi .
$$

The action of the quantum group on each subspace $\overline{\mu_{u}} \otimes H_{u}$ is the tensor product of the trivial action on the first factor and the representation $u$ on the second. This action is ergodic.

Most importantly, the linear functional $\omega$ on ${ }^{\circ} \mathcal{C}_{\mu}$ which annihilates each subspace $\overline{\mu_{u}} \otimes H_{u}, u \in \hat{G}, u \neq \iota$ and takes $I$ to 1 is a positive and faithful state; it is the unique state invariant under the action (i.e. $\omega \otimes$ id $\circ \alpha=\omega$ ). Therefore ${ }^{\circ} \mathcal{C}_{\mu}$ has a $C^{*}$-norm. It turns out that the maximal $C^{*}$-norm is finite. We thus have at our disposal two possible completions of ${ }^{\circ} \mathcal{C}_{\mu}$, which are different in general, the completion in the maximal $C^{*}$-norm, denoted $\mathcal{C}_{\mu}$, and the completion in the norm provided by the GNS representation $\pi_{\omega}$ of the invariant state, called the reduced completion.

The $G$-action clearly extends to the maximal completion. In the case of the reduced completion, note that the action of $G$ on ${ }^{\circ} \mathcal{C}_{\mu}$ is in fact only an action of the dense spectral Hopf ${ }^{*}$-subalgebra. This action extends to an action of the reduced compact quantum group $G_{\text {red }}$ and in turn it lifts to a normal action of the Hopf-von Neumann algebra generated by the regular representation of $G$ on the von Neumann algebra $\pi_{\omega}(\mathcal{C})^{\prime \prime}$. In all these cases, the extended action is ergodic; see Theorem 2.5 of 13 . 


\section{Modular theory of the invariant state}

In view of the duality theorem recalled in the previous section, we may and shall think of the spectral functor of an ergodic $C^{*}$-action of a compact quantum group $G$ as an abstract quasitensor functor $(\mu, \tilde{\mu}): \operatorname{Rep}(G) \rightarrow$ Hilb. Correspondingly, we shall represent the dense spectral subalgebra with generators and relations given in $(2.5)$.

If $a=\bar{k} \otimes \psi, b=\overline{k^{\prime}} \otimes \psi^{\prime}$ have support in the irreducible representations $u$ and $v$ respectively, then

$$
\begin{gathered}
\omega\left(a^{*} b\right)=\delta_{u, v}\left\|R_{u}\right\|^{-2}\left(k^{\prime}, J_{u}{ }^{*} J_{u} k\right)\left(\psi, \psi^{\prime}\right), \\
\omega\left(b a^{*}\right)=\delta_{u, v}\left\|\bar{R}_{u}\right\|^{-2}\left(k^{\prime}, k\right)\left(\psi,\left(j_{u}{ }^{*} j_{u}\right)^{-1} \psi^{\prime}\right) ;
\end{gathered}
$$

see Section 8 in 9 for explicit computations. These formulas may be used to derive modular properties of the invariant state, in turn generalizing the corresponding properties of the Haar state [15, 3]. In fact, for every irreducible representation $u$ choose a standard solution $\left(R_{u}, \bar{R}_{u}\right)$. We have a densely defined multiplicative map such that on $\overline{\mu_{u}} \otimes H_{u}$,

$$
\sigma_{-i}(\bar{k} \otimes \psi)=\overline{\left(J_{u}^{*} J_{u}\right) k} \otimes j_{u}^{*} j_{u} \psi
$$

with inverse

$$
\sigma_{i}(\bar{k} \otimes \psi)=\overline{\left(J_{u}^{*} J_{u}\right)^{-1} k} \otimes\left(j_{u}^{*} j_{u}\right)^{-1} \psi .
$$

Since a different standard solution is of the form $U j_{u}$, with $U$ unitary, the associated $J_{u}$ changes into $\mu(U) J_{u}$; hence $\sigma_{-i}$ and its inverse do not change. A quick computation shows that the KMS property holds,

$$
\omega\left(\sigma_{-i}(b) a^{*}\right)=\omega\left(a^{*} b\right) .
$$

We may choose $j_{\bar{u}}=j_{u}^{-1}$, which implies $J_{\bar{u}}=J_{u}^{-1}$. It follows that $\sigma_{-i}\left(a^{*}\right)=\sigma_{i}(a)^{*}$. We collect the conclusions of the above discussion, a slight refinement of a result of [15, [3].

Theorem 3.1. The invariant state of an ergodic action of a compact quantum group $G$ on a unital $C^{*}$-algebra satisfies the KMS condition on the dense spectral subalgebra. It is a trace if and only if for any spectral irreducible representation $u$ of $G$,

a) $d(u)=\operatorname{dim}(u)$, i.e. the antilinear $j_{u}$ defining a standard solution is antiunitary; and

b) the associated $J_{u}$ is antiunitary (hence $\left.q-\operatorname{mult}(u)=\operatorname{mult}(u)\right)$.

Recall that the condition $d(u)=\operatorname{dim}(u)$ for all $u$ means precisely that the quantum group is of Kac type; i.e. it has involutive coinverse. For example, quantum dimensions of the real deformations $G_{q}, 0<q<1$ of classical compact Lie groups are known to be strictly larger than the corresponding integral dimensions; hence the invariant state of any ergodic $C^{*}$-algebra under the action of any of these quantum groups is not a trace.

Almost periodicity of the invariant state. If $(\mathcal{C}, \alpha, G)$ is an ergodic action of a compact quantum group, the dense spectral subalgebra of $\mathcal{C}$ is the domain of a one-parameter group of ${ }^{*}$-automorphisms, the modular group, given by

$$
\sigma_{t}(\bar{k} \otimes \psi)=\overline{\left(J_{u}{ }^{*} J_{u}\right)^{i t} k} \otimes\left(j_{u}^{*} j_{u}\right)^{-i t} \psi .
$$


This group extends to a one-parameter automorphism group of the maximal completion $\mathcal{C}_{\mu}$. Moreover, it extends to $\pi_{\omega}\left(\mathcal{C}_{\mu}\right)$ or $M:=\pi_{\omega}\left(\mathcal{C}_{\mu}\right)^{\prime \prime}$ as well since it leaves $\omega$ invariant. In this subsection we only consider the extension to the von Neumann algebra $M$. Note that the cyclic vector $\Omega$ associated to the GNS representation of $\omega$ is separating for $M$. This may be shown with arguments similar to those of Section 4 of 6 . Hence, by the KMS property, $\sigma_{i}$ becomes the restriction of the modular operator $\Delta_{\omega}$ associated to $\omega$ under the canonical inclusion ${ }^{\circ} \mathcal{C}_{\mu} \rightarrow L^{2}(\mathcal{C}, \omega)$. Recall that Connes defined a normal faithful state $\phi$ on a von Neumann algebra to be almost periodic if $\Delta_{\phi}$ is diagonal. In the case of an ergodic action, the expressions for the inner product (3.1) and for $\sigma_{i},(3.3)$, show that the cyclic state of $M$ thus obtained is always almost periodic. Moreover, the point spectrum of $\Delta_{\omega}$ is completely determined by the eigenvalues of $j_{u}^{*} j_{u}$ and $J_{u}^{*} J_{u}$ for $u$ describing a complete set of irreducible spectral representations. While the spectrum of $j_{u}^{*} j_{u}$ is a structural property of the quantum group, that of $J_{u}^{*} J_{u}$ depends on the ergodic action, and this is the most mysterious part. Indeed, although $J_{u}$ is explicitly associated with $j_{u}$, we cannot infer that properties of $j_{u}$ pass to $J_{u}$. For example, we may have $j_{u}$ antiunitary for all spectral $u$ but $J_{u}$ not antiunitary, or, in other words, we may have ergodic actions of compact quantum groups of Kac type on a unital $C^{*}$-algebra $\mathcal{C}$ for which $M=\pi_{\omega}(\mathcal{C})^{\prime \prime}$ is a type III factor [13. Similarly, we may have $j_{u}$ not antiunitary for all spectral $u$ but $J_{u}$ always antiunitary, as in the examples arising from subfactors, described in detail in [10].

We conclude this section with a few more remarks on the modular theory of $(\mathcal{C}, \alpha, G)$. Let $\operatorname{Sp}\left(\Delta_{\omega}\right)\left(\operatorname{Sp}_{p}\left(\Delta_{\omega}\right)\right)$ denote the spectrum (point spectrum) of $\Delta_{\omega}$. The following fact may be known.

Theorem 3.2. If an ergodic $C^{*}$-action of a compact quantum group $G$ on $\mathcal{C}$ admits a spectral irreducible representation $u$ of $G$ such that $d(u)>\operatorname{dim}(u)$, then $\operatorname{Sp}_{p}\left(\Delta_{\omega}\right) \neq\{1\}$. If in addition both $M=\pi_{\omega}(\mathcal{C})^{\prime \prime}$ and the centralizer $M_{\omega}$ are factors, then $M$ is of type $\mathrm{III}_{\lambda}$ with $0<\lambda \leq 1$.

Proof. If $u$ is a spectral irreducible representation for which $j_{u}$ is not antiunitary, then $j_{u}^{*} j_{u}$ has an eigenvalue $<1$ and another $>1$ since $j_{u}$ is standard. Hence $\Delta_{\omega}$ has an eigenvalue $\neq 1$ on $\overline{\mu_{u}} \otimes H_{u}$. If $M$ and $M_{\omega}$ are factors, it is well known that $S(M)=\operatorname{Sp}\left(\Delta_{\omega}\right)$, with $S(M)$ the Connes invariant (Cor. 3.2.7 in [4]). Hence $S(M) \neq\{1\}$ and $S(M) \neq\{0,1\}$, so $M$ is not semifinite or of type $\mathrm{III}_{0}$.

In the next section we shall give general lower and upper bounds for the eigenvalues of the modular operator $\Delta_{\omega}$, depending only on the quantum group. We shall derive a general lower bound for the possible parameters $0<\lambda<1$ such that $M=\pi_{\omega}(\mathrm{C})^{\prime \prime}$ is a factor of type $\mathrm{III}_{\lambda}$ with a factorial centralizer $M_{\omega}$.

\section{An analogue of the finiteness theorem for CQG}

We have thus seen that the point spectrum of the modular operator $\Delta_{\omega}$ associated to an ergodic action is completely determined by the spectra of the positive matrices $\left(J_{u}^{*} J_{u}\right) \otimes\left(j_{u}^{*} j_{u}\right)$ associated to the spectral irreducible representations $u$ of the quantum group.

In this section we derive a general estimate for the eigenvalues of the operators $J_{u}^{*} J_{u}$. Our estimate involves the growth rate of the quantum dimension of $u$. It allows us to generalize the finiteness result of [7] to compact quantum groups. 
We define the growth rate of the intrinsic dimension of an object $u$ of a tensor $C^{*}$-category,

$$
\begin{gathered}
D_{u, n}:=\max \left\{d(v), \text { irreducible subobjects } v \text { of } u^{\otimes n}\right\}, \\
D_{u}:=\lim _{n}\left(D_{u, n}\right)^{1 / n} .
\end{gathered}
$$

This limit always exists since $D_{u, n}$ is a submultiplicative sequence. Note that $D_{u} \leq d(u), D_{u}=D_{v}$ if $u$ and $v$ are equivalent, and $D_{\bar{u}}=D_{u}$. We say that the intrinsic dimension of $u$ has subexponential (exponential) growth if $D_{u}=1$ $\left(D_{u}>1\right)$. Note that if $w$ is a subrepresentation of some tensor power $u^{\otimes k}$ of $u$, $D_{w} \leq D_{u}^{k}$. Hence, if the tensor powers of $u$ contain all the irreducibles and if $d(u)$ has subexponential growth, so does every irreducible.

Example 4.1. Let $G$ be a compact group. In this case the quantum dimension of any representation $u$ is just the integral dimension of the corresponding Hilbert space. It is known that this dimension always has polynomial, and hence subexponential, growth. This fact relies on Weyl's dimension formula and played an important role in the original proof of the finiteness theorem of [7].

Let $(\mathcal{C}, \alpha)$ be an ergodic action of a compact quantum group $G$ on a unital $C^{*}-$ algebra. We shall also need to consider the growth rate of integral multiplicities of irreducible representations of $G$ in $\mathcal{C}$. Given a representation $u$, set

$$
\begin{gathered}
\operatorname{Mult}_{u, n}:=\max \left\{\operatorname{mult}(v), \text { irreducible subrepresentations } v \text { of } u^{\otimes n}\right\}, \\
\operatorname{Mult}_{u}:=\liminf _{n} \operatorname{Mult}_{u, n}^{1 / n},
\end{gathered}
$$

which enjoys properties similar to those of $D_{u}$. In the particular case of the translation action of $G$ over itself, integral multiplicities mult $(u)$ reduce to integral dimensions $\operatorname{dim}(u)$. We shall accordingly denote the corresponding growth rate by $\operatorname{Dim}_{u}$ and refer to it as the growth rate of integral multiplicities. In both cases, we have the notion of subexponential or exponential growth. A comparison between the various growth rates introduced may be easily derived:

$$
\text { Mult }_{u} \leq D_{u}, \quad \operatorname{Dim}_{u} \leq D_{u} .
$$

Example 4.2. Let $G$ be a classical compact Lie group and $G_{q}$ the associated deformed compact matrix quantum group by a positive parameter $0<q<1$. The integral dimensions of irreducibles have subexponential growth as they are the same as in the classical case. On the other hand, the quantum dimensions of irreducibles have exponential growth rate. This last assertion is known. However, it may also be derived from the following proposition. Indeed, $D_{u}=1$ would imply $\lambda_{u}=\Lambda_{u}=1$, hence $j_{u}$ antiunitary.

Proposition 4.3. For any representation u of a compact quantum group,

$$
D_{u}^{-1} \leq \lambda_{u} \leq \Lambda_{u} \leq D_{u} .
$$

If $\operatorname{Dim}_{u}=1$, then the first and last inequalities are equalities.

Proof. The middle inequality being obvious, it suffices to show that $\Lambda_{u} \leq D_{u}$, as $\lambda_{u} \geq D_{u}^{-1}$ follows passing to the conjugate representation. The $n$-th tensor product solution $j_{u \otimes n}=\left(j_{u} \otimes \cdots \otimes j_{u}\right) \theta_{n}$ for $u^{\otimes n}$, with $\theta_{n}$ a suitable permutation operator, 
is standard if $j_{u}$ is. If $v$ is an irreducible subrepresentation of $u^{\otimes n}$ and $j_{v}$ is a standard solution for $v, d(v)=\operatorname{Trace}\left(j_{v}^{*} j_{v}\right) \geq \Lambda_{v}$. Hence

$$
\begin{aligned}
D_{u, n} & \geq \max \left\{\Lambda_{v}, v \text { irreducible subrepresentation of } u^{\otimes n}\right\} \\
& =\Lambda_{u} \otimes n \\
& =\Lambda_{u}^{n} .
\end{aligned}
$$

On the other hand, $d(v) \leq \Lambda_{v} \operatorname{dim}(v)$ implies

$$
D_{u, n} \leq \Lambda_{u \otimes n} \operatorname{Dim}_{u, n}=\Lambda_{u}^{n} \operatorname{Dim}_{u, n} ;
$$

hence $D_{u} \leq \Lambda_{u}$ if the integral dimension of $u$ has subexponential growth rate.

Remark. This proposition may be used to explain why the spectrum of the associated matrices $j_{u}^{*} j_{u}$ has a symmetric shape for the deformed compact matrix quantum groups $G_{q}$.

For the Wang-Van Daele quantum groups $A_{o}(F)$ and $A_{u}(F)$, we follow the notation of [1]. We shall always normalize $F$ so that $\operatorname{Trace}\left(F^{*} F\right)=\operatorname{Trace}\left(\left(F^{*} F\right)^{-1}\right)$. Recall that for $A_{o}(F)$, the matrix $F$ is required to satisfy $F \bar{F}= \pm 1$.

Example 4.4. If $u$ is the fundamental representation of $A_{u}(F)$, with $\operatorname{rank}(F) \geq 2$, all tensor powers $u^{\otimes n}$ are irreducible [1, showing that $D_{u, n}=d(u)^{n}$ by multiplicativity of quantum dimension. Hence $D_{u}=d(u)=\operatorname{Trace}\left(F^{*} F\right)$ is the largest possible value. Similarly, $\operatorname{Dim}_{u}=\operatorname{dim}(u)$. In particular, the extreme inequalities in Proposition 4.3 are always strict (the first examples being $A_{u}(m), \lambda_{u}=\Lambda_{u}=1$ but $D_{u}=\operatorname{Dim}_{u}=m$ ), while the middle inequality is generically strict for $\operatorname{rank}(F) \geq 3$, but it is an equality for $\operatorname{rank}(F)=2$ due to $\operatorname{Trace}\left(F^{*} F\right)=\operatorname{Trace}\left(\left(F^{*} F\right)^{-1}\right)$.

Example 4.5. If $u_{1}$ is the fundamental representation of $G=S_{q} U(2)$, for a nonzero $|q| \leq 1,|q|^{1 / 2} R=\psi_{1} \otimes \psi_{2}-q \psi_{2} \otimes \psi_{1}$ is a standard solution; hence $j_{u_{1}}^{*} j_{u_{1}}=\operatorname{diag}\left(|q|,|q|^{-1}\right)$. Let $u_{r}$ be the unique irreducible $(r+1)$-dimensional representation. The Clebsch-Gordan rule $u_{1} \otimes u_{r} \simeq u_{r-1} \oplus u_{r+1}$ gives $j_{u_{r}}^{*} j_{u_{r}}=$ $\operatorname{diag}\left(|q|^{r},|q|^{r-2}, \ldots,|q|^{-r}\right)$. Hence $\lambda_{u_{r}}=\Lambda_{u_{r}}^{-1}=D_{u_{r}}^{-1}=|q|^{r}$.

Example 4.6. Consider the quantum group $A_{o}(F)$. It is well known that the representation categories of $A_{o}(F)$ and $S_{\mp q} U(2)$ are tensor equivalent if $q>0$ is defined by $q+\frac{1}{q}=\operatorname{Trace}\left(F^{*} F\right)$. Hence $D_{u_{r}}^{A_{o}(F)}=q^{-r}$ by Example 4.5. Note that $\lambda_{u_{r}}=\Lambda_{u_{r}}^{-1}$. This follows from the validity for $r=1$ and the Clebsch-Gordan rule. A computation shows that for every $F$ and for the fundamental representation $u_{1}$, the extreme inequalities in Proposition 4.3 are strict iff $\operatorname{rank}(F) \geq 3$.

Example 4.7. For $\operatorname{rank}(F) \geq 3$, the integral dimension of the fundamental representation $u_{1}$ of $A_{o}(F)$ has exponential growth. This may be seen in the following way. Independently of the matrix $F$, irreducible representations of $A_{o}(F)$ satisfy the same fusion rules as those of $S U(2)$ [1]. Hence, denoting with the same symbol the corresponding irreducible representations, the integral dimensions are determined by the Clebsch-Gordan rule, $\operatorname{dim}\left(u_{r+1}\right)=\operatorname{dim}\left(u_{1}\right) \operatorname{dim}\left(u_{r}\right)-\operatorname{dim}\left(u_{r-1}\right) \geq$ $2 \operatorname{dim}\left(u_{r}\right)$; hence $\operatorname{Dim}_{u_{1}, r}=\operatorname{dim}\left(u_{r}\right) \geq 2^{r-1} \operatorname{dim}\left(u_{1}\right)$.

The following is our main result. 
Theorem 4.8. For any spectral irreducible representation $u$ of an ergodic action of a compact quantum group on a unital $C^{*}$-algebra,

a) $D_{u}^{-1} \leq J_{u}^{*} J_{u} \leq D_{u}$, where $J_{u}$ is associated to a standard solution $j_{u}$ for $u$.

b) If the spectral functor is a relaxed tensor and if Mult $_{u}=1$, then $\frac{1}{D_{u}}$ and $D_{u}$ are respectively the smallest and the largest eigenvalues of $J_{u}^{*} J_{u}$.

Proof. a) The first inequality follows from the second applied to $\bar{u}$. By Lemma 2.2a), for any positive integer $n$, if $J_{u}$ is associated to any solution $j_{u}$ for $u$,

$$
\begin{aligned}
& \left\|J_{u}^{*} J_{u}\right\|^{n}=\left\|J_{u}^{*} J_{u} \otimes \cdots \otimes J_{u}^{*} J_{u}\right\| \\
& \quad=\left\|\tilde{\mu}_{u, \ldots, u}^{*} J_{u}^{* \otimes n} J_{u \otimes n} \tilde{\mu}_{u, \ldots, u}\right\| \leq\left\|J_{u \otimes n}^{*} J_{u \otimes n}\right\|,
\end{aligned}
$$

where $J_{u \otimes n}$ is associated to the $n$-th tensor product solution $j_{u \otimes n}=\left(j_{u} \otimes \cdots \otimes j_{u}\right) \theta_{n}$ for $u^{\otimes n}$. Consider a complete reduction of $u^{\otimes n}$ into a direct sum of irreducible representations $v$ and let $S_{v} \in\left(v, u^{\otimes n}\right)$ be the isometry associated to $v$. We may compute $\left\|J_{u \otimes n}^{*} J_{u \otimes n}\right\|$ as the norm of the positive operator-valued matrix $\left(\mu\left(S_{v}^{*}\right) J_{u \otimes n}^{*} J_{u}^{\otimes n} \mu\left(S_{w}\right)\right)_{v, w}$. By Lemma 2.2b), $\mu\left(j_{u}^{\otimes n} S_{v} j_{v}^{-1}\right) J_{v}=J_{u \otimes n} \mu\left(S_{v}\right)$, with respect to any solution $j_{v}$ for $v$, with associated $J_{v}$. Now we fix standard solutions for $u$ and all the $v$. Since $j_{u} \otimes n$ is standard, we may find $\left\{S_{v}\right\}$ such that $j_{u \otimes n} S_{v} j_{v}^{-1}=: \bar{S}_{v}$ are pairwise orthogonal isometries. Hence

$$
\mu\left(S_{v}^{*}\right) J_{u \otimes n}^{*} J_{u \otimes n} \mu\left(S_{w}\right)=J_{v}^{*} \mu\left(\bar{S}_{v}^{*} \bar{S}_{w}\right) J_{w}=\delta_{v, w} J_{v}^{*} J_{w} .
$$

Combination with the previous estimate gives

$$
\begin{aligned}
& \left\|J_{u}^{*} J_{u}\right\| \leq\left\|\operatorname{diag}_{v}\left(J_{v}^{*} J_{v}\right)\right\|^{1 / n}=\left(\max _{v}\left\{\left\|J_{v}^{*} J_{v}\right\|\right\}\right)^{1 / n} \\
& \quad \leq\left(\max _{v}\left\{\operatorname{Trace}\left(J_{v}^{*} J_{v}\right)\right\}\right)^{1 / n}=\left(\max _{v}\left\{\left\|\hat{R}_{v}\right\|^{2}\right\}\right)^{1 / n} \leq\left(\max _{v}\left\{\left\|R_{v}\right\|^{2}\right\}\right)^{1 / n}=D_{u, n}^{1 / n} .
\end{aligned}
$$

b) If the spectral functor is a relaxed tensor, all the $\tilde{\mu}$ are unitary; hence the first and the last inequalities are equalities. On the other hand, the estimate $\max _{v}\left\{\operatorname{Trace}\left(J_{v}^{*} J_{v}\right)\right\} \leq \max _{v}\left\{\left\|J_{v}^{*} J_{v}\right\|\right\} \mathrm{Mult}_{u, n}$ shows that $\left\|J_{u}^{*} J_{u}\right\|=D_{u}$ if the integral multiplicity of $u$ has subexponential growth.

Corollary 4.9 ([7]). The invariant state of an ergodic action of a compact group on a unital $C^{*}$-algebra is a trace.

Proof. As recalled above, for all $u, D_{u, n}$ has polynomial growth. Hence the $J_{u}$ are all antiunitary by Theorem 4.8. On the other hand, the same holds in the classical case for standard solutions $j_{u}$ of $G$-respresentations; hence we may apply Theorem 3.1.

We next discuss examples satisfying b) with $D_{u}>1$ and which are not translation actions.

Example 4.10. Let $G_{q}$ be as above a deformed classical compact Lie group, and let $\Phi: \operatorname{Rep}\left(G^{\prime}\right) \rightarrow \operatorname{Rep}\left(G_{q}\right)$ be a tensor equivalence, with $G^{\prime}$ another compact matrix quantum group (e.g. $G=S U(2)$ and $G^{\prime}=A_{o}(F)$ ). The composition $\mu: \operatorname{Rep}\left(G^{\prime}\right) \rightarrow \operatorname{Rep}\left(G_{q}\right) \rightarrow$ Hilb with the embedding functor of $\operatorname{Rep}\left(G_{q}\right)$ is a relaxed tensor functor. Consider the maximal ergodic action associated to $\mu$. The integral multiplicity of an irreducible representation $u$ of $G^{\prime}$ is the integral dimension of $\Phi(u)$, which is the same as in the classical case; hence $\operatorname{Mult}_{u}^{G^{\prime}}=\operatorname{Dim}_{\Phi(u)}^{G_{q}}=1$. Note that these are just the inverses of the tensor equivalences of [2]. 
Corollary 4.11. If a compact quantum group $G$ of Kac type admits an ergodic action on a unital $C^{*}$-algebra with non-tracial invariant state, then the integral dimension of some spectral irreducible representation of $G$ has exponential growth.

Proof. Since $G$ has involutive coinverse, every standard solution $j_{u}$ is antiunitary. If all the quantum dimensions of spectral irreducibles $u$ had subexponential growth, the associated $J_{u}$ would be antiunitary by Theorem 4.8 ; hence the invariant state would be a trace by Theorem 3.1.

Corollary 4.12. $S_{-1} U(d)$ acts ergodically only on tracial $C^{*}$-algebras.

Corollary 4.13. Let $G$ be a compact quantum group acting ergodically on $\mathcal{C}$ and admitting a spectral irreducible representation $u$ s.t. $d(u)>\operatorname{dim}(u)$. Assume that $M:=\pi_{\omega}(\mathcal{C})^{\prime \prime}$ and $M_{\omega}$ are factors.

a) If $M$ is of type $\mathrm{III}_{\lambda}, 0<\lambda<1$, then

$$
\lambda \geq \sup \left\{\frac{\min \left\{\lambda_{u}, \Lambda_{u}^{-1}\right\}}{D_{u}}, u \text { spectral irreducible s.t. } d(u)>\operatorname{dim}(u)\right\},
$$

b) if the above supremum is $1, M$ is of type $\mathrm{III}_{1}$.

Proof. a) Let $u$ be a spectral irreducible representation such that $d(u)>\operatorname{dim}(u)$. As argued in Theorem 3.2, the modular group of $\omega$ does not act trivially on the spectral subspace $\overline{\mu_{u}} \otimes H_{u}$. On the other hand, the smallest and largest eigenvalues of the restriction of the modular operator $\Delta_{\omega}$ to $\overline{\mu_{u}} \otimes H_{u}$ are bounded below by $\frac{1}{D_{u} \Lambda_{u}}$ and above by $\frac{D_{u}}{\lambda_{u}}$. Taking into consideration the fact that the eigenvalues of $\Delta_{\omega}$ belong to $S(M)$, we see that either $\frac{1}{D_{u} \Lambda_{u}} \leq \lambda$ or $\lambda^{-1} \leq \frac{D_{u}}{\lambda_{u}}$.

b) If the supremum is 1 , by a), $M$ is not of type $\mathrm{III}_{\lambda}$ for any $0<\lambda<1$. Hence $M$ must be of type $\mathrm{III}_{1}$ by Theorem 3.2.

Remark. If the quantum group is of Kac type, under the same assumptions of the previous corollary, we similarly derive $\lambda \geq \frac{1}{n}$, with $n$ the minimal dimension of a spectral irreducible representation for which the restriction of the modular group on the associated spectral subspace is non-trivial.

Example 4.14. The lower bound in the above remark is realized in Wang's example of ergodic action of $A_{u}(n)$ on the type $\mathrm{III}_{\underline{1}}$ factors [13. More precisely, in this case the factor is the von Neumann completion of the Cuntz algebra $\mathcal{O}_{n}$ in the GNS representation of the canonical state and the modular group is the group describing the $\mathbb{Z}$-gradation. This automorphism group acts non-trivially on the generating Hilbert space of isometries. This Hilbert space carries the fundamental representation of the quantum group. This example shows that $D_{u}$, together with triviality of the modular theory of $A_{u}(n)$, explain completely the factorial type.

Remark. If $\lambda_{u}=D_{u}^{-1}=\Lambda_{u}{ }^{-1}$, e.g. under the condition of Proposition 4.3, the lower bound for $\lambda$ becomes simply $\sup \left\{\lambda_{u}^{2}, u: d(u)>\operatorname{dim}(u)\right\}$. In particular, for $S_{q} U(2)$, with $0<|q|<1$, under the same assumptions of the previous theorem, the possible parameters $\lambda$ satisfy $\lambda \geq|q|^{2 r}$, where $r$ is the smallest strictly positive integer such that $u_{r}$ is spectral. Hence, for a given $q$, small values of $\lambda$ are possible only if the spectrum of the action has gaps.

The following example shows that the assumption of subexponential growth of integral multiplicities cannot be removed in Theorem $4.8 \mathrm{~b}$ ). 
Example 4.15. For $A_{o}(F)$, with $F$ of rank $\geq 3$, taking into account Example 4.6 and $\lambda_{u_{r}}=\Lambda_{u_{r}}^{-1}=\|F\|^{-2 r}$, we see that $\lambda \geq\left(\frac{q}{\|F\|^{2}}\right)^{r}$, where $q$ is defined by $\operatorname{Trace}\left(F^{*} F\right)=\operatorname{Trace}\left(\left(F^{*} F\right)^{-1}\right)=q+\frac{1}{q}$ and $u_{r}$ is again the first spectral irreducible representation. In particular, consider the translation action of $A_{o}(F)$ over itself. Vaes and Vergnioux have shown, among other things, that if $\operatorname{Trace}\left(F^{*} F\right) \geq \sqrt{5}\|F\|^{2}$, then the Haar state $h$ is factorial and the associated von Neumann algebra is a full factor. Moreover, if the spectrum of $\left(F^{*} F\right)^{-1} \otimes F^{*} F$ generates the subgroup $\left\{\lambda^{n}, n \in \mathbb{Z}\right\}$, for some $0<\lambda<1, \pi_{h}\left(A_{o}(F)\right)^{\prime \prime}$ is a factor of type $\mathrm{III}_{\lambda}$ [12]. The fundamental representation is spectral. In this example our lower bound $\lambda \geq \frac{q}{\|F\|^{2}}$ is not optimal already for $F^{*} F=\operatorname{diag}\left(\lambda, 1, \lambda^{-1}\right)$.

\section{ACKNOWLEDGEMENT}

The author would like to thank Alessandro Figà-Talamanca for a conversation related to this paper.

\section{REFERENCES}

[1] T. Banica: Le groupe quantique compact libre U(n). Comm. Math. Phys., 190 (1997), 143-172. MR1484551 (99k:46095)

[2] J. Bichon, A. De Rijdt, S. Vaes: Ergodic coactions with large multiplicity and monoidal equivalence of quantum groups. Comm. Math. Phys., 262 (2006), 703-728. MR2202309 (2007a:46072)

[3] F.P. Boca: Ergodic actions of compact matrix pseudogroups on $C^{*}$-algebras. In: Recent advances in operator algebras (Orléans, 1992). Astérisque, 232 (1995), 93-109. MR.1372527 (97d:46075)

[4] A. Connes: Une classification des facteurs de type III. Ann. Sci. École Norm. Sup., 6 (1973), 133-252. MR0341115(49:5865)

[5] A. Connes: Almost periodic states and factors of type $\mathrm{III}_{1}$. J. Funct. Analysis, 16 (1974), 415-445. MR0358374 (50:10840)

[6] S. Doplicher, R. Longo, J.E. Roberts, L. Zsido: A remark on quantum group actions and nuclearity. Rev. Math. Phys., 14 (2002), 787-796. MR1932666 (2003h:46097)

[7] R. Høegh-Krohn, M.B. Landstad, E. Størmer: Compact ergodic groups of automorphisms, Ann. of Math. (2), 114 (1981), 75-86. MR625345 (82i:46097)

[8] R. Longo, J.E. Roberts: A theory of dimension. K-Theory, 11 (1997), 103-159. MR1444286 (98i:46065)

[9] C. Pinzari, J.E. Roberts: A duality theorem for ergodic actions of compact quantum groups on $C^{*}$-algebras. Comm. Math. Phys., 277 (2008), 385-421. MR2358289 (2008k:46203)

[10] C. Pinzari, J.E. Roberts: Ergodic actions of $S_{\mu} U(2)$ on $C^{*}$-algebras from $\mathrm{II}_{1}$ subfactors. J. Geom. Phys., 60 (2010), 403-416. MR2600003 (2011f:46084)

[11] C. Pinzari, J.E. Roberts: A theory of induction and classification of tensor $C^{*}$-categories, J. Noncomm. Geom., to appear, arXiv:0907.2459.

[12] S. Vaes, R. Vergnioux: The boundary of universal discrete quantum groups, exactness, and factoriality. Duke Math. J., 140 (2007), 35-84. MR2355067(2010a:46166)

[13] S. Wang: Ergodic actions of universal quantum groups on operator algebras. Comm. Math. Phys., 203 (1999), 481-498. MR.1697607 (2000j:46128)

[14] A. Wassermann: Ergodic actions of compact groups on operator algebras. III. Classification for SU(2). Invent. Math., 93 (1988), 309-354. MR948104(91e:46093)

[15] S.L. Woronowicz: Compact matrix pseudogroups. Comm. Math. Phys., 111 (1987), 613665. MR.901157 (88m:46079)

[16] S.L. Woronowicz: Compact quantum groups. In: Symétries quantiques (Les Houches, 1995), 845-884, North-Holland, Amsterdam, 1998. MR1616348 (99m:46164)

Dipartimento di Matematica, Sapienza Università di Roma, 00185-Roma, Italy

E-mail address: pinzari@mat.uniroma1.it 